\title{
Adaptive Differentiator-Based Predefined-Time Control for Nonlinear Systems Subject to Pure-Feedback Form and Unknown Disturbance
}

\author{
Man Yang ${ }^{D},{ }^{1}$ Qiang Zhang, ${ }^{1}$ Ke Xu, ${ }^{1}$ and Ming Chen ${ }^{2}$ \\ ${ }^{1}$ School of Mathematical Sciences, Bohai University, Jinzhou 121000, Liaoning, China \\ ${ }^{2}$ School of Electronic and Information Engineering, University of Science and Technology Liaoning, Anshan 114000, \\ Liaoning, China
}

Correspondence should be addressed to Man Yang; yangman@qymail.bhu.edu.cn

Received 20 May 2021; Accepted 15 July 2021; Published 28 July 2021

Academic Editor: Xiaodi Li

Copyright (c) 2021 Man Yang et al. This is an open access article distributed under the Creative Commons Attribution License, which permits unrestricted use, distribution, and reproduction in any medium, provided the original work is properly cited.

In this article, by utilizing the predefined-time stability theory, the predefined-time output tracking control problem for perturbed uncertain nonlinear systems with pure-feedback structure is addressed. The nonaffine structure of the original system is simplified as an affine form via the property of the mean value theorem. Furthermore, the design difficulty from the uncertain nonlinear function is overcome by the excellent approximation performance of RBF neural networks (NNs). An adaptive predefined-time controller is designed by introducing the finite-time differentiator which is used to decrease the computational complexity problem appeared in the traditional backstepping control. It is proved that the proposed control method guarantees all signals in the closed-loop system remain bound and the tracking error converges to zero within the predefined time. Based on the controller designed in this paper, the expected results can be obtained in predefined time, which can be illustrated by the simulation results.

\section{Introduction}

Nonlinear control systems with uncertain parameters are widely existed in practical applications, which can be dealt with effectively by the adaptive backstepping control [1]. The idea of backstepping is to construct the Lyapunov function step by step according to the system, and the corresponding virtual controller is deduced to obtain the control law in the final step [2]. The main advantage of the adaptive backstepping method is that the considered control systems do not require the matching conditions. The research on backstepping-based adaptive control for nonlinear systems in strict-feedback form attracted increasing attention in the control field and many interesting results have been published in [3-7], such as the results on SISO nonlinear systems [3-5] and the results on MIMO nonlinear systems [6, 7]. In addition, by combining neural network (NN) $[8,9]$ with adaptive backstepping design method, many control methods were proposed for nonlinear systems [10-12].
Many models of real systems are pure-feedback systems such as robots [13], spacecrafts [14], and engineering machinery [15]. In order to deal with the stability problem of pure-feedback system, many effective control schemes have been proposed. For example, the authors in [16] simplify the nonlinear pure-feedback system using the implicit function theorem, and then an improved controller was obtained. Using the small-gain theorem, an ISS-modular approach for nonlinear systems with pure-feedback structure was created in [17] and an improved controller with a novel weight estimator was constructed to solve the problems in controlling the nonaffine pure-feedback system. In addition, in [18], based on the mean value theorem, the problem that arises from the structure of nonlinear pure-feedback system is settled by an adaptive neural control.

All of the abovementioned control methods achieve the control objectives when time goes to infinity. It is obvious that, in the system for practical application, we would like the system to be stable in finite time. Therefore, it is very 
meaningful to study the finite-time control (FTC) method. The related theory of finite-time stability can be found in literature studies $[19,20]$, which is put forward by P. Dorato and L. Weiss et al. Since then, the design of finite-time controller has attracted the attention of many researchers, and a lot of relevant results have been presented. In [21], the stability of strict-feedback nonlinear systems with time delay and quantization was investigated for the first time by combining finite-time theory, neural networks, and adaptive control methods, and an improved barrier Lyapunov function (BLF) was constructed. Mehdi Golestani et al. [22] designed a finite-time robust controller using the fast terminal sliding mode approach, in which a bipolar sigmoid function is proposed to alleviate chattering phenomenon. In [23], a finite-time global stabilizer was designed for uncertain nonlinear systems, which guarantees global finitetime stabilization of Hölder continuous nonlinear systems. Based on the properties of fuzzy logic system, an effective adaptive fault-tolerant control method was received in [24], which effectively addressed the stability problem of the system with actuator faults. More recently, the authors in [25] introduced an improved finite-time controller to deal with the problems of nonlinear systems with unknown hysteresis. In all of the FTC results presented above, the convergence time is related to the initial value of the system states, which is going to be long when the initial states are far away from the equilibrium $x=0$. So, in order to let the convergence time in finite-time control be independent of the initial value of the system states, the concept of fixedtime control was proposed.

Fixed-time stability was proposed by Polyakov, and then he systematically elaborated the difference between finitetime stability and fixed-time stability in [26]. According to fixed-time stability theory, the settling time to stabilize nonlinear system is a constant, which has no relation with the initial states. Furthermore, the fixed-time controller can effectively improve the transient performance of the control system, and many improved fixed-time controllers have been proposed. For example, two novel fixed-time controllers were applied to the nonlinear systems in [27, 28], which take advantage of the approximate performance of fuzzy logic system and the backstepping method. Besides, fixed-time stability of delayed static neural networks was concerned in [29]. In [30], a fixed-time adaptive eventtriggered tracking controller of uncertain nonlinear systems was proposed. In addition, by combining the characteristics of the barrier Lyapunov function with the backstepping technique, a new controller was introduced in [31] for the nonlinear system with multi-input and multioutput uncertainties, and the convergence time of this control strategy is a fixed constant. In spite of having the meaningful advantage over finite-time stability, fixed-time stability theory cannot give an exact expression of the convergence time according to the design parameters. Thus, in order to overcome this shortcoming, the predefined-time stability theorem was derived by Sánchez-Torres et al. in [32], which can give the expression of the convergence time function according to the design controller parameters. The predefined-time theory has been applied to various types of systems by plenty of researchers, and a large number of meaningful research results have emerged subsequently. Recently, the advantages of predefined-time theory were applied to second-order multi-intelligent systems [33] and nonlinear mechanical systems [34]. In [35], according to the characteristic of predefined-time stability theory, an improved predefined-time adaptive control structure was proposed to deal with the system with dead zone. Furthermore, in [36], based on sliding mode control technology, a predefined-time controller was designed for second-order nonlinear systems. Although many improved predefinedtime controllers have been presented, it is difficult to design a predefined-time controller for the nonlinear pure-feedback system due to the nonaffine structure; therefore, we need to design a predefined-time control scheme for the nonlinear pure-feedback system with unknown disturbance.

Inspired by the aforementioned discussions, an advanced adaptive predefined-time control scheme was constructed for pure-feedback nonlinear system with unknown disturbances, and the expected performance was achieved within a predefined time. Based on the above discussions, the innovations of this article are listed as follows:

(i) The theory of predefined time was firstly applied to $n$-order nonlinear pure-feedback system. Then, an NN-based adaptive predefined-time controller was firstly used for nonlinear systems in pure-feedback form with unknown disturbance, too.

(ii) Based on the mean value theorem, the simplified system structure is obtained, which can reduce the complexity of the controller design. Furthermore, the derivatives of the virtual controllers are estimated by finite-time differentiators in this work, which avoids the issue of explosion of complexity.

The rest of this article is organized as follows. In Section 2 , the nonlinear pure-feedback system is simplified by mean value theorem, and the relevant preliminary knowledge is presented. The design process of the controller is elaborately described in Section 3 and the stability analysis of the system is elaborately described in Section 4. In Section 5, the effectiveness of the designed controller is illustrated by the simulation results. Finally, Section 6 summarizes this article.

\section{System Formulation and Preliminaries}

2.1. System Formulation. A nonlinear pure-feedback system with unknown disturbance is considered as follows:

$$
\left\{\begin{array}{l}
\dot{x}_{i}(t)=f_{i}\left(\bar{x}_{i}, x_{i+1}\right), \quad 1 \leq i \leq n-1, \\
\dot{x}_{n}(t)=f_{n}\left(\bar{x}_{n}, u\right)+d(t) \\
y(t)=x_{1}(t)
\end{array}\right.
$$

in which $\bar{x}_{i}(t)=\left[x_{1}(t), \ldots, x_{i}(t)\right]^{T} \in R^{i}, i=1, \ldots, n$ is the vector of the states; $y(t) \in R$ represents the system output; $u \in R$ denotes the input signal; $d(t)$ is an unknown bounded disturbance and satisfies $|d(t)| \leq \bar{d}$ with $\bar{d}$ being a constant; and $f_{i}(\cdot), i=1, \ldots, n$ represents unknown smooth functions. 
The nonaffine structure of the original system (1) can be simplified by the property of the mean value theorem [18].
Alternative expressions for the unknown smooth functions $f_{i}(\cdot), i=1, \ldots, n$ in system (1) are shown as follows:

$$
\begin{aligned}
f_{i}\left(\bar{x}_{i}, x_{i+1}\right) & =f_{i}\left(\bar{x}_{i}, x_{i+1}^{0}\right)+\left.\frac{\partial f_{i}\left(\bar{x}_{i}, x_{i+1}\right)}{\partial x_{i+1}}\right|_{x_{i+1}=x_{\mu_{i}}} \times\left(x_{i+1}-x_{i+1}^{0}\right), \quad 1 \leq i \leq n-1, \\
f_{n}\left(\bar{x}_{n}, u\right) & =f_{n}\left(\bar{x}_{n}, u^{0}\right)+\left.\frac{\partial f_{n}\left(\bar{x}_{n}, u\right)}{\partial u}\right|_{u=x_{\mu_{n}}} \times\left(u-u^{0}\right),
\end{aligned}
$$

where $\quad x_{\mu_{i}}=\mu_{i} x_{i+1}+\left(1-\mu_{i}\right) x_{i+1}^{0}$, with $0<\mu_{i}<1$, $i=1, \ldots, n-1$, and $x_{\mu_{n}}=\mu_{n} u+\left(1-\mu_{n}\right) u^{0}$, with $0<\mu_{n}<1$.

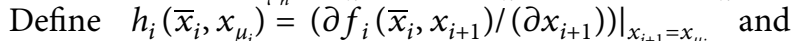
$h_{n}\left(\bar{x}_{n}, x_{\mu_{n}}\right)=\left(\partial f_{n}\left(\bar{x}_{n}, u\right)\right) /\left.(\partial u)\right|_{u=x_{u}}$, which are unknown nonlinear functions. After that, substituting (2) and (3) into system (1) and choosing $x_{i+1}^{0}=0, u^{0}=0$, one has

$$
\left\{\begin{array}{l}
\dot{x}_{i}(t)=f_{i}\left(\bar{x}_{i}\right)+h_{i}\left(\bar{x}_{i}, x_{\mu_{i}}\right) x_{i+1}, \\
\dot{x}_{n}(t)=f_{n}\left(\bar{x}_{n}\right)+h_{n}\left(\bar{x}_{n}, x_{\mu_{n}}\right) u+d(t), \\
y(t)=x_{1}(t)
\end{array}\right.
$$

The aim of this paper is to design a predefined-time adaptive controller for system (1), which can ensure all signals in the closed-loop system are bounded, while the tracking error converges to zero within a predefined time. The following assumptions and lemmas are provided to ease the controller design.

Assumption 1 [37]: the function $h_{i}\left(\bar{x}_{i}, x_{\mu_{i}}\right)$ satisfies $0<\underline{h}_{i} \leq h_{i}(\cdot) \leq \bar{h}_{i}<\infty$, for $i=1, \ldots, n$, where $\underline{h}_{i}$ and $\bar{h}_{i}$ are unknown constants.

Assumption 2 [35]: the trajectory signal $y_{r}$ is a continuous and bounded function. Usually, the time derivatives of $y_{r}$ is also continuous and bounded function. One can find constants $A_{1}, A_{2}$ such that $\left|y_{r}\right| \leq A_{1},\left|\dot{y}_{r}\right| \leq A_{2}$, but $A_{1}$ and $A_{2}$ are unknown.

2.2. On Predefined-Time Stability. Consider the following system:

$$
\begin{aligned}
\dot{x} & =h(x, \rho), \\
x_{0} & =x(0),
\end{aligned}
$$

where $x \in R^{n}$ and $\rho \in R^{b}$ denote the state and the parameter of system (5), respectively; $h: R^{n} \longrightarrow R^{n}$ stands for nonlinear function and $h(0, \rho)=0 ; x_{0}=x(0) \in D$ represents initial state of system (5) and suppose that the equilibrium of system (5) is the origin $x=0$.

Definition 1 (see [38]). The origin is a fixed-time stable equilibrium of system (5), if it is finite-time stable and $T\left(x_{0}\right)$ is bounded on $R^{n}$, i.e., $\exists T_{\text {max }}>0, \forall x_{0} \in R^{n}, T\left(x_{0}\right) \leq T_{\text {max }}$.

Definition 2 (predefined-time stability [32, 39]). If the constant $T_{\max }$ given in Definition 1 is a function of the system parameter $\rho$, i.e., $T_{\max }=T_{\max }(\rho)$, the origin of system (5) is weak predefined-time stable. Furthermore, if the time $T_{\max }$ is also a minimum setting time bound, the origin of system (5) is strong predefined-time stable.

Definition 3 (see [40]).

$$
\operatorname{sig}(x)^{\rho}=\left[\left|x_{1}\right|^{\rho} \operatorname{sign}\left(x_{1}\right), \ldots,\left|x_{n}\right|^{\rho} \operatorname{sign}\left(x_{n}\right)\right]^{T},
$$

for $x=\left[x_{1}, x_{2}, \ldots, x_{n}\right]^{T} \in R^{n}$, where $\rho>0$ and $\operatorname{sign}(\cdot)$ denotes the standard signum function.

\subsection{Mathematical Lemmas}

Lemma 1 (see [32, 41]). If there exists a continuous radially unbounded function $V: R^{n} \longrightarrow R_{+} \cup\{0\}$, such that $V(x)=0$ for $x \in G$ and any solution $x(t)$ satisfies

$$
\dot{V} \leq \frac{-\beta}{\rho T} \exp \left(\frac{1}{\beta}(2 V)^{(\rho / 2)}\right)(2 V)^{1-(\rho / 2)},
$$

for $0<\beta$ and $0<\rho<2$, the set $G$ is globally predefined-time attractive for system (5); then $T_{\max }=T$.

Proof. By solving the above differential inequality, the following inequality can be obtained:

$$
V(x) \leq \frac{1}{2}\left[\beta \ln \left(\frac{T}{t+T \exp \left(-(1 / \beta)\left(2 V_{0}\right)^{(\rho / 2)}\right)}\right)\right]^{-(\rho / 2)},
$$

where $V_{0}=V\left(x_{0}\right)$. It is a fact that $V(x)=0$ if $T /\left(t+T \exp \left(-(1 / \beta)\left(2 V_{0}\right)^{(\rho / 2)}\right)\right)=1$, and thus, the convergence time function is

$$
T\left(x_{0}\right)=T\left(1-\exp \left(-\frac{1}{\beta}\left(2 V_{0}\right)^{(\rho / 2)}\right)\right) .
$$

Then, $T_{\max }=T$ since $1-\exp \left(-(1 / \beta)\left(2 V_{0}\right)^{(\rho / 2)}\right) \leq 1$.

Lemma 2 (see [3]). Let $x_{q}=\left[x_{1}, x_{2}, \ldots, x_{q}\right]^{T}$ and $S\left(\bar{x}_{q}\right)=$ $\left[s_{1}\left(\bar{x}_{q}\right), \ldots, s_{l}\left(\bar{x}_{q}\right)\right]^{T}$ is the basis function vector. Then, for any positive integer $m \leq q$, we have

$$
\left\|S\left(\bar{x}_{q}\right)\right\|^{2} \leq\left\|s\left(\bar{x}_{m}\right)\right\|^{2} .
$$

Lemma 3 (see [35]). Suppose two similarly ordered sequences $\quad a=\left(a_{1}, \ldots, a_{n}\right)$ and $b=\left(b_{1}, \ldots, b_{n}\right) \quad$ satisfy 
$a_{1} \leq \cdots \leq a_{n}, b_{1} \leq \cdots \leq b_{n}$ or $a_{1} \geq \cdots \geq a_{n}, b_{1} \geq \cdots \geq b_{n}$, then we have

$$
\sum_{i=1}^{n} a_{i} b_{i} \geq \frac{1}{n} \sum_{i=1}^{n} a_{i} \sum_{i=1}^{n} b_{i}
$$

Lemma 4 (see [42]). For $X=\left[x_{1}, \ldots, x_{l}\right]^{T}, Y=\left[y_{1}, \ldots\right.$, $\left.y_{l}\right]^{T}$, one has

$$
\begin{aligned}
& \left|x_{1} y_{1}+x_{2} y_{2}+\cdots+x_{l} y_{l}\right| \\
& \leq \sqrt{x_{1}^{2}+x_{2}^{2}+\cdots+x_{l}^{2}} \sqrt{y_{1}^{2}+y_{2}^{2}+\cdots+y_{l}^{2}} \\
& \leq\|X\|\|Y\| .
\end{aligned}
$$

Lemma 5 (see [35]). For positive real sequence $a=\left(a_{1}\right.$, $\left.\ldots, a_{n}\right)$, the following inequality holds:

$$
\left(\prod_{i=1}^{n} a_{i}\right)^{(1 / n)} \leq \frac{1}{n} \sum_{i=1}^{n} a_{i}
$$

Lemma 6 (see [35]). Suppose $\kappa_{i} \in R$ and $0<l \leq 1$, then

$$
\sum_{i=1}^{m}\left|\kappa_{i}\right|^{l} \geq\left(\sum_{i=1}^{m}\left|\kappa_{i}\right|\right)^{l}
$$

The universal approximation performance of radial basis function neural networks (RBF NN) can be utilized to estimate the unknown function $f(\xi)$, and the approximation function is given as follows:

$$
f(\xi)=W^{T} \Phi(\xi)+\delta(\xi), \quad \delta(\xi)<\varepsilon,
$$

where $\xi \in \Omega_{\xi} \subset R^{m}$ is the input vector and $W=\left[w_{1}, w_{1}, \ldots, w_{n}\right]$ represents the weight vector; $\delta(\xi)$ is an approximation error and $\varepsilon>0$ expresses the accuracy level; and $\Phi(\xi)=\left[\varphi_{1}(\xi), \varphi_{2}(\xi), \ldots, \varphi_{n}(\xi)\right]$ denotes the basis function vector with the NN node number $n>1$. In addition, $\varphi_{i}(\xi)$ is the Gaussian function which is written as follows:

$$
\varphi_{i}(\xi)=\exp \left(-\frac{\left(\xi-\zeta_{i}\right)^{T}\left(\xi-\zeta_{i}\right)}{\eta_{i}^{T} \eta_{i}}\right), \quad i=1,2, \ldots, n,
$$

where $\zeta_{i}=\left[\zeta_{i 1}, \zeta_{i 1}, \ldots, \zeta_{i m}\right]$ is the center of the receptive field and $\eta_{i}=\left[\eta_{i 1}, \eta_{i 1}, \ldots, \eta_{i m}\right]$ denotes the width of the Gaussian function.

Lemma 7 (see [37]). A continuous function $f(\xi)$ on a compact set $\Omega_{\xi} \subset R^{m}$ can be approximated by RBF NN (15) if the node number $n$ is sufficiently large, and

$$
f(\xi)=W^{* T} \Phi(\xi)+\delta(\xi), \quad \delta(\xi)<\varepsilon,
$$

where $W^{*}$ is the ideal weight vector and defined by

$$
W^{*}=\underset{W \in R^{m}}{\arg \min }\left\{\sup _{\xi \in \Omega_{\xi}}\left|f(\xi)-W^{T} \varphi(\xi)\right|\right\} \text {. }
$$

\section{Controller Design}

In this section, an adaptive predefined-time tracking controller will be designed for system (4) via backstepping technique. In the design process, we will use the following coordinate transformation:

$$
\begin{aligned}
z_{1} & =y-y_{r}, \\
z_{i} & =x_{i}-\alpha_{i-1}, \quad i=2, \ldots, n,
\end{aligned}
$$

where $\alpha_{(i-1)}, i=2, \ldots, n$ is the virtual control signal for the $(i-1)$ th subsystem, $y_{r}$ represents the trajectory function, and $z_{1}$ denotes the tracking error. Then the main design procedure of this paper is given as follows.

Remark 1. In the process of controller design, define $f_{i}=f_{i}\left(\bar{x}_{i}\right), i=1, \ldots, n, h_{i}=h_{i}\left(\bar{x}_{i}, x_{\mu_{i}}\right), i=1, \ldots, n-1$, and $h_{n}=h_{n}\left(\bar{x}_{n}, x_{\mu_{n}}\right)$.

Step 1. A Lyapunov function is constructed as $V_{1}=(1 / 2) z_{1}^{2}$. Then, based on systems (4) and (19), we can get

$$
\begin{aligned}
\dot{z}_{1} & =\dot{y}-\dot{y}_{r}=\dot{x}_{1}-\dot{y}_{r}=f_{1}+h_{1} x_{2}-\dot{y}_{r} \\
& =f_{1}+h_{1}\left(z_{2}+\alpha_{1}\right)-\dot{y}_{r},
\end{aligned}
$$

where $x_{2}=z_{2}+\alpha_{1}$.

Differentiating $V_{1}$ results in

$$
\begin{aligned}
\dot{V}_{1} & =z_{1} \dot{z}_{1} \\
& =z_{1}\left(f_{1}+h_{1}\left(z_{2}+\alpha_{1}\right)-\dot{y}_{r}\right) \\
& =z_{1}\left(f_{1}-\dot{y}_{r}\right)+z_{1} h_{1} z_{2}+z_{1} h_{1} \alpha_{1} .
\end{aligned}
$$

The universal approximation performance of RBF NN can be utilized to estimate the unknown function $f_{1}$, and the approximation function is given as follows:

$$
f_{1}=\widehat{W}_{1}^{T} \Phi_{1}\left(\bar{x}_{1}\right)+\delta_{1}\left(\bar{x}_{1}\right) .
$$

Then, according to Lemmas 2 and 4, we can obtain

$$
\begin{aligned}
f_{1}-\dot{y}_{r} & =\widehat{W}_{1}^{T} \Phi_{1}\left(\bar{x}_{1}\right)+\delta_{1}\left(\bar{x}_{1}\right)-\dot{y}_{r} \\
& \leq \widehat{W}_{1}^{T} \Phi_{1}\left(\bar{x}_{1}\right)+\delta_{M 1}+A_{1} \\
& \leq\left\|\bar{W}_{1}\right\|\left\|\Phi_{1}\right\| \leq \sqrt{q+2}\left\|\bar{W}_{1}\right\| \\
& \leq \frac{1}{2}\left(q+2+\left\|\bar{W}_{1}\right\|^{2}\right),
\end{aligned}
$$

where $\widehat{W}_{1} \in R^{q}$ is the RBF NN weight vector, $\Phi_{1}\left(\bar{x}_{1}\right)=$ $\left[\Phi_{11}\left(\bar{x}_{1}\right), \ldots, \Phi_{1 q}\left(\bar{x}_{1}\right)\right]^{T}$ denotes the basis function vector, $\delta_{1}(\bar{x})$ is the approximation error and satisfies $\delta_{1}\left(\bar{x}_{1}\right) \leq \delta_{M 1}$ with $\delta_{M 1}$ being a constant, and $\bar{W}_{1}=\left[\widehat{W}_{1}, \delta_{M 1}, A_{1}\right]$, $\bar{\Phi}_{1}=\left[\Phi_{1}\left(\bar{x}_{1}\right), 1,1\right]^{T}$.

Let $\theta_{1}=\left\|\bar{W}_{1}\right\|^{2}$ and $\widetilde{\theta}_{1}=\theta_{1}-\widehat{\theta}_{1}$, where $\widehat{\theta}_{1}$ is the estimation of $\theta_{1}$. Subsequently, using Young's inequality, the following inequality can be obtained:

$$
z_{1}\left(f_{1}-\dot{y}_{r}\right) \leq \frac{1}{2}\left(q+2+\theta_{1}\right)\left|z_{1}\right| .
$$


Substituting (24) into (21), we have

$$
\begin{aligned}
\dot{V}_{1} & \leq \frac{1}{2}\left(q+2+\widehat{\theta}_{1}+\widetilde{\theta}_{1}\right)\left|z_{1}\right|+z_{1} h_{1} z_{2}+z_{1} h_{1} \alpha_{1} \\
& \leq \frac{1}{2}\left(q+2+\widehat{\theta}_{1}\right)\left|z_{1}\right|+\frac{1}{2} \widetilde{\theta}_{1}\left|z_{1}\right|+z_{1} h_{1} z_{2}+z_{1} h_{1} \alpha_{1} .
\end{aligned}
$$

The unknown parameter $\tilde{\theta}_{1}$ can be solved by defining a Lyapunov function $W_{1}$ as follows:

$$
W_{1}=V_{1}+\frac{1}{2} \widetilde{\theta}_{1}^{2}
$$

Differentiating $W_{1}$ results in

$$
\begin{aligned}
\dot{W}_{1} & =\dot{V}_{1}-\tilde{\theta}_{1} \dot{\hat{\theta}}_{1} \\
& \leq \frac{1}{2}\left(q+2+\widehat{\theta}_{1}\right)\left|z_{1}\right|+\frac{1}{2}\left|z_{1}\right| \widetilde{\theta}_{1}+z_{1} h_{1} z_{2}+z_{1} h_{1} \alpha_{1}-\widetilde{\theta}_{1} \dot{\hat{\theta}}_{1} \\
& \leq \frac{1}{2}\left(q+2+\widehat{\theta}_{1}\right)\left|z_{1}\right|+z_{1} h_{1} z_{2}+z_{1} h_{1} \alpha_{1}+\widetilde{\theta}_{1}\left(\frac{1}{2}\left|z_{1}\right|-\dot{\hat{\theta}}_{1}\right) .
\end{aligned}
$$

Now, we design the feasible virtual control $\alpha_{1}$ as

$$
\alpha_{1}=\frac{-1}{\underline{h}_{1}}\left(\frac{n}{\rho T} \operatorname{sig}\left(z_{1}\right)^{1-\rho} \exp \left(\left|z_{1}\right|^{\rho}\right)+\frac{1}{2}\left(\widehat{\theta}_{1}+q+2\right) \operatorname{sign}\left(z_{1}\right)+\lambda_{1} \operatorname{sign}\left(z_{1}\right)\right),
$$

where $T$ is the predefined time, $q$ is the hidden neuron number, $n$ is the NN node number, $\lambda_{1}$ is a positive tuning parameter which will be given later, and $\rho$ is the design parameter which satisfies $0<\rho<2$.

Remark 2. By selecting the value of the designed parameter $\lambda_{1}$ in the virtual controller $\alpha_{1}$, we can make sure that $(1 / 2) \tilde{\theta}_{1}-\lambda_{1}$ is a negative number. follows:

The adaptive law for the parameter $\hat{\theta}_{1}$ is designed as

$$
\begin{aligned}
\dot{\hat{\theta}}_{1} & =\frac{1}{2}\left|z_{1}\right|, \\
\dot{\hat{\theta}}_{1}(0) & >0 .
\end{aligned}
$$

Then, we have

$$
\dot{W}_{1} \leq \frac{-n}{\rho T}\left|z_{1}\right|^{2-\rho} \exp \left(\left|z_{1}\right|^{\rho}\right)+z_{1} h_{1} z_{2}-\lambda_{1}\left|z_{1}\right| \text {. }
$$

Step 2. In order to overcome the obstacle of finding the firstorder derivative of the virtual controller, an effective approximation tool called finite-time differentiator is brought out with the following structure:

$$
\left\{\begin{array}{l}
\dot{\sigma}_{11}=\sigma_{12}-k_{1} \operatorname{sig}\left(\sigma_{11}-\alpha_{1}\right)^{(1 / 2)} \\
\dot{\sigma}_{12}=-k_{2} \operatorname{sign}\left(\sigma_{11}-\alpha_{1}\right)
\end{array}\right.
$$

where $\sigma_{11}$ and $\sigma_{12}$ represent the states of differentiator and $k_{1}$ and $k_{2}$ are differentiator parameters. According to [10, 43], the boundedness of $\sigma_{11}(0)-\alpha_{1}(0)$ and $\sigma_{12}(0)-\dot{\alpha}_{1}(0)$ ensure that the finite-time differentiator can estimate the firstorder derivative of the virtual controller with arbitrary accuracy. Thus, another alternative form of the first-order derivative of the virtual controller is $\dot{\alpha}_{1}(t)=\sigma_{12}(t)+\varepsilon_{1}$, where $\varepsilon_{1}$ denotes the bounded estimation error, and a constant $\varepsilon_{M 1}>0$ can be found such that $\varepsilon_{1}$ satisfies the inequality $\left|\varepsilon_{1}\right| \leq \varepsilon_{M 1}$.

The dynamic of tracking error $z_{2}=x_{2}-\alpha_{1}$ satisfies

$$
\dot{z}_{2}=f_{2}+h_{2}\left(z_{3}+\alpha_{2}\right)-\dot{\alpha}_{1} \text {, }
$$

where $z_{3}=x_{3}-\alpha_{2}$.

Design the Lyapunov function as

$$
V_{2}=V_{1}+\frac{1}{2} z_{2}^{2}
$$

Differentiating $V_{2}$ results in

$$
\begin{aligned}
\dot{V}_{2} & =\dot{V}_{1}+z_{2}\left(f_{2}-\dot{\alpha}_{1}\right)+z_{2} h_{2} z_{3}+z_{2} h_{2} \alpha_{2} \\
& =\dot{V}_{1}+z_{2}\left(f_{2}-\dot{\alpha}_{1}+\sigma_{12}\right)-z_{2} \sigma_{12}+z_{2} h_{2} z_{3}+z_{2} h_{2} \alpha_{2} \\
& =\dot{V}_{1}+z_{2}\left(f_{2}+\varepsilon_{1}\right)-z_{2} \sigma_{12}+z_{2} h_{2} z_{3}+z_{2} h_{2} \alpha_{2},
\end{aligned}
$$

where $\varepsilon_{1}=\sigma_{12}-\dot{\alpha}_{1}$. The universal approximation performance of RBF NN can be utilized to estimate the unknown function $f_{2}$, and the approximation function is given as follows:

$$
f_{2}=\widehat{W}_{2}^{T} \Phi_{2}\left(\bar{x}_{2}\right)+\delta_{2}\left(\bar{x}_{2}\right) .
$$

Then, according to Lemmas 2 and 4, we can obtain

$$
\begin{aligned}
f_{2}+\varepsilon_{1} & =\widehat{W}_{2}^{T} \Phi_{2}\left(\bar{x}_{2}\right)+\delta_{2}\left(\bar{x}_{2}\right)+\varepsilon_{1} \\
& \leq\left(\widehat{W}_{2}^{T} \Phi_{2}\left(\bar{x}_{2}\right)+\delta_{M 2}\left(\bar{x}_{2}\right)+\varepsilon_{M 1}\right) \\
& \leq\left\|\bar{W}_{2}\right\|\left\|\bar{\Phi}_{2}\right\| \leq \sqrt{q+2}\left\|\bar{W}_{2}\right\| \\
& \leq \frac{1}{2}\left(q+2+\left\|\bar{W}_{2}\right\|^{2}\right) \\
& \leq \frac{1}{2}\left(q+2+\theta_{2}\right),
\end{aligned}
$$

where $\widehat{W}_{2} \in R^{q}$ is the RBF NN weight vector, $\Phi_{2}\left(\bar{x}_{2}\right)=$ $\left[\Phi_{21}\left(\bar{x}_{2}\right), \ldots, \Phi_{2 q}\left(\bar{x}_{2}\right)\right]^{T}$ denotes the basis function vector, and $\delta_{2}(\bar{x})$ is the approximation error and satisfies $\delta_{2}\left(\bar{x}_{2}\right) \leq \delta_{M 2}$ with $\delta_{M 2}$ being a constant; besides, we define $\bar{W}_{2}=\left[\widehat{W}_{2}, \delta_{M 2}, \varepsilon_{M 1}\right]^{T}, \bar{\Phi}_{2}=\left[\Phi_{2}\left(\bar{x}_{2}\right), 1,1\right]^{T}$, and let $\theta_{2}=\left\|\bar{W}_{2}\right\|^{2}, \theta_{2}=\theta_{2}-\widehat{\theta}_{2}$, where $\widehat{\theta}_{2}$ is the estimation of $\theta_{2}$. 
Substituting (36) into (34) and using Young's inequality give

$$
\begin{aligned}
\dot{V}_{2} & \leq \dot{V}_{1}+\frac{1}{2}\left|z_{2}\right|\left(q+2+\theta_{2}\right)-z_{2} \sigma_{12}+z_{2} h_{2} z_{3}+z_{2} h_{2} \alpha_{2} \\
& \leq \dot{V}_{1}+\frac{1}{2}\left|z_{2}\right|\left(q+2+\widehat{\theta}_{2}\right)+\frac{1}{2}\left|z_{2}\right| \widetilde{\theta}_{2}-z_{2} \sigma_{12}+z_{2} h_{2} z_{3}+z_{2} h_{2} \alpha_{2} .
\end{aligned}
$$

The unknown parameter $\tilde{\theta}_{2}$ can be solved by defining a Lyapunov function $W_{2}$ as follows:

$$
W_{2}=V_{2}+\frac{1}{2} \widetilde{\theta}_{2}^{2}
$$

Then, differentiating $W_{2}$ results in

$$
\begin{aligned}
& \dot{W}_{2}=\dot{V}_{2}-\widetilde{\theta}_{2} \dot{\hat{\theta}}_{2} \\
& \leq \dot{V}_{1}+\frac{1}{2}\left(q+2+\widehat{\theta}_{2}\right)\left|z_{2}\right|+\frac{1}{2}\left|z_{2}\right| \widetilde{\theta}_{2}-z_{2} \sigma_{12}+z_{2} h_{2} z_{3}+z_{2} h_{2} \alpha_{2}-\widetilde{\theta}_{2} \dot{\hat{\theta}}_{2} \\
& \leq \dot{V}_{1}+\frac{1}{2}\left(q+2+\widehat{\theta}_{2}\right)\left|z_{2}\right|-z_{2} \sigma_{12}+z_{2} h_{2} z_{3}+z_{2} h_{2} \alpha_{2}+\widetilde{\theta}_{2}\left(\frac{1}{2}\left|z_{2}\right|-\dot{\hat{\theta}}_{2}\right) . \\
& \quad \dot{\sigma}_{(i-1) 1}=\sigma_{(i-1) 2}-k_{1} \operatorname{sig}\left(\sigma_{(i-1) 1}-\alpha_{i-1}\right)^{(1 / 2)}, \\
& \dot{\sigma}_{(i-1) 2}=-k_{2} \operatorname{sign}\left(\sigma_{(i-1) 1}-\alpha_{i-1}\right),
\end{aligned}
$$

$$
\begin{aligned}
\alpha_{2}= & -\frac{1}{\underline{h}_{2}}\left(\frac{n}{\rho T} \operatorname{sig}\left(z_{2}\right)^{1-\rho} \exp \left(\left|z_{2}\right|^{\rho}\right)+\frac{1}{2}\left(\hat{\theta}_{2}+q+2\right) \operatorname{sign}\left(z_{2}\right)\right. \\
& \left.+\left|\sigma_{12}\right| \operatorname{sign}\left(z_{2}\right)+\lambda_{2} \operatorname{sign}\left(z_{2}\right)+\left|z_{1}\right| \bar{h}_{1} \operatorname{sign}\left(z_{2}\right)\right),
\end{aligned}
$$

where $T$ is the predefined time, $q$ is the hidden neuron number, $\lambda_{2}$ is a positive tuning parameter which will be given later, and the parameter $\rho$ satisfies $0<\rho<2$.

The adaptive law for the parameter $\widehat{\theta}_{2}$ is designed as follows:

$$
\begin{aligned}
\dot{\hat{\theta}}_{2} & =\frac{1}{2}\left|z_{2}\right|, \\
\dot{\hat{\theta}}_{2}(0) & >0 .
\end{aligned}
$$

Then we can rewrite (37) in the following form:

$$
\dot{V}_{2} \leq \sum_{i=1}^{2}\left(\frac{-n}{\rho T}\left|z_{i}\right|^{2-\rho} \exp \left(\left|z_{i}\right|^{\rho}\right)+\left(\frac{1}{2} \widetilde{\theta}_{i}-\lambda_{i}\right)\left|z_{i}\right|\right)+z_{2} h_{2} z_{3}
$$

and we can get

$$
\dot{W}_{2} \leq \sum_{i=1}^{2}\left(\frac{-n}{\rho T}\left|z_{i}\right|^{2-\rho} \exp \left(\left|z_{i}\right|^{\rho}\right)-\lambda_{i}\left|z_{i}\right|\right)+z_{2} h_{2} z_{3} .
$$

Step $i . \quad(3 \leq i \leq n-1)$. In order to overcome the obstacle of finding the first-order derivative of the virtual controller $\alpha_{i-1}$, an effective approximation tool called finite-time differentiator is brought out with the following structure: where $\sigma_{(i-1) 1}$ and $\sigma_{(i-1) 2}$ represent the states of differentiator and $k_{1}$ and $k_{2}$ denote differentiator parameters. Differentiator (44) can produce an approximate function of $\dot{\alpha}_{i-1}$ with any precision only if the initial deviations $\sigma_{(i-1) 1}(0)-$ $\alpha_{i-1}(0)$ and $\sigma_{(i-1) 2}(0)-\dot{\alpha}_{i-1}(0)$ are bounded. Thus, another alternative form of the first-order derivative of the virtual controller is $\dot{\alpha}_{i-1}(t)=\sigma_{(i-1) 2}(t)+\varepsilon_{i-1}$, where $\varepsilon_{i-1}$ denotes the bounded estimation error, and we can find a positive constant $\varepsilon_{M(i-1)}$ such that the inequality $\left|\varepsilon_{i-1}\right| \leq \varepsilon_{M(i-1)}$ holds.

The dynamic of tracking error $z_{i}=x_{i}-\alpha_{i-1}$ satisfies

$$
\dot{z}_{i}=f_{i}+h_{i}\left(z_{i+1}+\alpha_{i}\right)-\dot{\alpha}_{i-1} \text {. }
$$

The universal approximation performance of RBF NN can be utilized to estimate the unknown function $f_{i}$, and the approximation function is given as follows:

$$
f_{i}=\widehat{W}_{i}^{T} \Phi_{i}\left(\bar{x}_{i}\right)+\delta_{i}\left(\bar{x}_{i}\right)
$$

where $\widehat{W}_{i} \in R^{q}$ is the $\mathrm{RBF} \mathrm{NN}$ weight vector, $\Phi_{i}(\bar{x})=\left[\Phi_{i 1}(\bar{x}), \ldots, \Phi_{i q}(\bar{x})\right]^{T}$ denotes the basis function vector, and $\delta_{i}(\bar{x})$ is the approximation error and satisfies $\delta_{i}(\bar{x}) \leq \delta_{M i}$ with $\delta_{M i}$ being a constant.

We design the feasible virtual control $\alpha_{i}$ as

$$
\begin{aligned}
\alpha_{i}= & -\frac{1}{\underline{h}_{i}}\left(\frac{n}{\rho T} \operatorname{sig}\left(z_{i}\right)^{1-\rho} \exp \left(\left|z_{i}\right|^{\rho}\right)\right. \\
& +\frac{1}{2}\left(\widehat{\theta}_{i}+q+2\right) \operatorname{sign}\left(z_{i}\right)+\left|\sigma_{(i-1) 2}\right| \operatorname{sign}\left(z_{i}\right) \\
& \left.+\lambda_{i} \operatorname{sign}\left(z_{i}\right)+\left|z_{i-1}\right| \bar{h}_{i-1} \operatorname{sign}\left(z_{i}\right)\right),
\end{aligned}
$$


where $T$ is the predefined time, $q$ is the hidden neuron number, $\lambda_{i}$ is a positive tuning parameter which will be designed later, and the parameter $\rho$ satisfies $0<\rho<2$.

The dynamic adaptive law for the unknown parameter is

$$
\begin{aligned}
\dot{\hat{\theta}}_{i} & =\frac{1}{2}\left|z_{i}\right|, \\
\dot{\hat{\theta}}_{i}(0) & >0 .
\end{aligned}
$$

The Lyapunov function is structured as follows:

$$
V_{i}=V_{i-1}+\frac{1}{2} z_{i}^{2}
$$

The time derivative of $V_{i}$ is obtained as follows:

$$
\begin{aligned}
\dot{V}_{i} & =\dot{V}_{i-1}+z_{i}\left(f_{i}+h_{i} x_{i+1}+\sigma_{(i-1) 2}-\dot{\alpha}_{i-1}\right)-z_{i} \sigma_{(i-1) 2} \\
& =\dot{V}_{i-1}+z_{i}\left(f_{i}+\sigma_{(i-1) 2}-\dot{\alpha}_{i-1}\right)-z_{i} \sigma_{(i-1) 2}+z_{i} h_{i} x_{i+1} \\
& \leq \dot{V}_{i-1}+z_{i}\left(f_{i}+\varepsilon_{M(i-1)}\right)-z_{i} \sigma_{(i-1) 2}+z_{i} h_{i}\left(z_{i+1}+\alpha_{i}\right) \\
& \leq \dot{V}_{i-1}+z_{i}\left(\widehat{W}_{i}^{T} \Phi_{i}\left(\bar{x}_{i}\right)+\delta_{i}\left(\bar{x}_{i}\right)+\varepsilon_{M(i-1)}\right)-z_{i} \sigma_{(i-1) 2}+z_{i} h_{i}\left(z_{i+1}+\alpha_{i}\right),
\end{aligned}
$$

where $x_{i+1}=z_{i+1}+\alpha_{i}$.

Then, according to Lemmas 2 and 4 and (46), we can have

$$
\begin{aligned}
f_{i}+\varepsilon_{M(i-1)} & =\widehat{W}_{i}^{T} \Phi_{i}\left(\bar{x}_{i}\right)+\delta_{i}\left(\bar{x}_{i}\right)+\varepsilon_{M(i-1)} \\
& \leq\left(\widehat{W}_{i}^{T} \Phi_{i}\left(\bar{x}_{i}\right)+\delta_{M i}\left(\bar{x}_{i}\right)+\varepsilon_{M(i-1)}\right) \\
& \leq\left\|\bar{W}_{i}\right\|\left\|\bar{\Phi}_{i}\right\| \leq \sqrt{q+2}\left\|\bar{W}_{i}\right\| \\
& \leq \frac{1}{2}\left(q+2+\left\|\bar{W}_{i}\right\|^{2}\right),
\end{aligned}
$$

where $\bar{W}_{i}=\left[\widehat{W}_{i}, \delta_{M i}, \varepsilon_{M i}\right]^{T}$ and $\bar{\Phi}_{i}=\left[\Phi_{i}\left(x_{i}\right), 1,1\right]^{T}$.

Employing Young's inequality leads to the following inequality:

$$
z_{i}\left(f_{i}+\varepsilon_{M(i-1)}\right) \leq \frac{1}{2}\left(q+2+\theta_{i}\right)\left|z_{i}\right|,
$$

where $\theta_{i}=\left\|\bar{W}_{i}\right\|^{2}, \tilde{\theta}_{i}=\theta_{i}-\widehat{\theta}_{i}$, and $\widehat{\theta}_{i}$ is the estimation of $\theta_{i}$.

Substituting (47) and (51) into (50), we have

$$
\begin{aligned}
& \dot{V}_{i} \leq \sum_{j=1}^{i}\left(\frac{-n}{\rho T}\left|z_{j}\right|^{2-\rho} \exp \left(\left|z_{j}\right|^{\rho}\right)+\left(\frac{1}{2} \widetilde{\theta}_{j}-\lambda_{j}\right)\left|z_{j}\right|\right)+z_{i} h_{i} z_{i+1}, \\
& \dot{W}_{i} \leq \sum_{j=1}^{i}\left(\frac{-n}{\rho T}\left|z_{j}\right|^{2-\rho} \exp \left(\left|z_{j}\right|^{\rho}\right)-\lambda_{j}\left|z_{j}\right|\right)+z_{i} h_{i} z_{i+1} .
\end{aligned}
$$

Step $n$. Similar to Step $i$, we construct the following differentiator to estimate $\dot{\alpha}_{n-1}$ :

$$
\left\{\begin{array}{l}
\dot{\sigma}_{(n-1) 1}=\sigma_{(n-1) 2}-k_{1} \operatorname{sig}\left(\sigma_{(n-1) 1}-\alpha_{(n-1)}\right){ }^{(1 / 2)}, \\
\dot{\sigma}_{(n-1) 2}=-k_{2} \operatorname{sign}\left(\sigma_{(n-1) 1}-\alpha_{n-1}\right),
\end{array}\right.
$$

where $\sigma_{(n-1) 1}$ and $\sigma_{(n-1) 2}$ represent the states of differentiator and $k_{1}$ and $k_{2}$ denote the differentiator parameters. Similar to the reason of Step $i$, another alternative form of the firstorder derivative of the virtual controller $\alpha_{n-1}$ is $\dot{\alpha}_{n-1}(t)=\sigma_{(n-1) 2}(t)+\varepsilon_{n-1}$, where $\varepsilon_{n-1}$ denotes the bounded estimation error, and a positive constant $\varepsilon_{M(n-1)}$ satisfying $\left|\varepsilon_{n-1}\right| \leq \varepsilon_{M(n-1)}$ can be found.

The dynamic of $z_{n}$ satisfies

$$
\begin{aligned}
\dot{z}_{n} & =\dot{x}_{n}-\dot{\alpha}_{n-1} \\
& =f_{n}+h_{n} u+d(t)-\dot{\alpha}_{n-1} .
\end{aligned}
$$

The real control input is chosen as follows:

$$
\begin{aligned}
u= & -\frac{1}{\underline{h}_{n}}\left(\frac{n}{\rho T} \operatorname{sig}\left(z_{n}\right)^{1-\rho} \exp \left(\left|z_{n}\right|^{\rho}\right)\right. \\
& +\frac{1}{2}\left(\widehat{\theta}_{n}+q+3\right) \operatorname{sign}\left(z_{n}\right)+\left|\sigma_{(n-1) 2}\right| \operatorname{sign}\left(z_{n}\right) \\
& \left.+\lambda_{n} \operatorname{sign}\left(z_{n}\right)+\left|z_{n-1}\right| \bar{h}_{n-1} \operatorname{sign}\left(z_{n}\right)\right),
\end{aligned}
$$

where $T$ is the predefined time, $q$ is the hidden neuron number, $\lambda_{n}$ is a positive tuning parameter which will be given later, and the parameter $\rho$ satisfies $0<\rho<2$.

The dynamic of the adaptive parameters is

$$
\begin{aligned}
\dot{\hat{\theta}}_{n} & =\frac{1}{2}\left|z_{n}\right|, \\
\dot{\hat{\theta}}_{i}(0) & >0 .
\end{aligned}
$$

The Lyapunov function is structured as follows:

$$
V_{n}=V_{n-1}+\frac{1}{2} z_{n}^{2}
$$

Differentiating $V_{n}$ leads to 


$$
\begin{aligned}
\dot{V}_{n} & =z_{n} \cdot \dot{z}_{n}+\dot{V}_{n-1}=z_{n}\left(f_{n}+h_{n} u-\dot{\alpha}_{n-1}+d(t)\right)+\dot{V}_{n-1} \\
& =\dot{V}_{n-1}+z_{n}\left(f_{n}-\dot{\alpha}_{n-1}+d(t)+\sigma_{(n-1) 2}\right)-z_{n} \sigma_{(n-1) 2}+z_{n} h_{n} u
\end{aligned}
$$

The universal approximation performance of RBF NN can be utilized to estimate the unknown function $f_{n}$, and the approximation function is given as follows:

$$
f_{n}=\widehat{W}_{n}^{T} \Phi_{n}(\bar{x})+\delta_{n}(\bar{x})
$$

where $\widehat{W}_{n} \in R^{q}$ is the $\mathrm{RBF}$ NN weight vector, $\Phi_{n}(\bar{x})=\left[\Phi_{n 1}(\bar{x}), \ldots, \Phi_{n q}(\bar{x})\right]^{T}$ denotes the basis function vector, and $\delta_{n}(\bar{x})$ is the approximation error and satisfies $\delta_{n}(\bar{x}) \leq \delta_{M n}$ with $\delta_{M n}$ being a constant.

Let $\bar{W}_{n}=\left[\bar{W}_{n}, \delta_{M n}, \varepsilon_{M n}, \bar{d}\right]^{T}, \bar{\Phi}_{n}=\left[\Phi_{n}(\bar{x}), 1,1,1\right]^{T}$, and RBF NN is introduced to approximate the unknown nonlinear function $f_{n}$. Then, using Lemmas 2 and 4 , we get

$$
\begin{aligned}
& f_{n}+\sigma_{(n-1) 2}-\dot{\alpha}_{n-1}+d(t) \\
& =\widehat{W}_{n}^{T} \Phi_{n}(\bar{x})+\delta_{n}(\bar{x})+\sigma_{(n-1) 2}-\dot{\alpha}_{n-1}+d(t) \\
& \leq \widehat{W}_{n}^{T} \Phi_{n}(\bar{x})+\delta_{M n}+\varepsilon_{M n}+\bar{d} \\
& \leq\left\|\bar{W}_{n}\right\|\left\|\bar{\Phi}_{n}\right\| \leq \frac{1}{2} \sqrt{q+3}\left\|\bar{W}_{n}\right\| .
\end{aligned}
$$

Utilizing Young's inequality, we get

$$
z_{n}\left(f_{n}(\bar{x})+\sigma_{(n-1) 2}-\dot{\alpha}_{n-1}+d(t)\right) \leq \frac{1}{2}\left(q+3+\theta_{n}\right)\left|z_{n}\right|,
$$

where $\theta_{n}=\left\|\bar{W}_{n}\right\|^{2}, \widetilde{\theta}_{n}=\theta_{n}-\widehat{\theta}_{n}$, and $\widehat{\theta}_{n}$ is the estimation of $\theta_{n}$.

Substituting (56) and (62) into (59), we have

$$
\begin{aligned}
\dot{V}_{n} & \leq \dot{V}_{n-1}+z_{n}\left(f_{n}-\dot{\alpha}_{n-1}+d(t)+\sigma_{(n-1) 2}\right)-z_{n} \sigma_{(n-1) 2}+z_{n} h_{n} u \\
& \leq \dot{V}_{n-1}+\frac{1}{2}\left(q+3+\theta_{n}\right)\left|z_{n}\right|-z_{n} \sigma_{(n-1) 2}+z_{n} h_{n} u \\
& \leq \sum_{i=1}^{n}\left(-\frac{n}{\rho T}\left|z_{i}\right|^{2-\rho} \exp \left(\left|z_{i}\right|^{\rho}\right)+\frac{1}{2} \sum_{i=1}^{n} \tilde{\theta}_{i}\left|z_{i}\right|-\sum_{i=1}^{n} \lambda_{i}\left|z_{i}\right|\right) .
\end{aligned}
$$

The unknown parameter $\tilde{\theta}_{n}$ can be solved by designing a Lyapunov function as follows:

$$
W_{n}=V_{n}+\frac{1}{2} \sum_{i=1}^{n} \tilde{\theta}_{i}^{2}
$$

Differentiating $W_{n}$ results in

$$
\begin{aligned}
\dot{W}_{n} & =\dot{V}_{n}-\sum_{i=1}^{n} \widetilde{\theta}_{i} \dot{\hat{\theta}}_{i} \\
& \leq \sum_{i=1}^{n}\left(-\frac{n}{\rho T}\left|z_{i}\right|^{2-\rho} \exp \left(\left|z_{i}\right|^{\rho}\right)+\frac{1}{2} \sum_{i=1}^{n} \widetilde{\theta}_{i}\left|z_{i}\right|-\sum_{i=1}^{n} \lambda_{i}\left|z_{i}\right|\right)-\sum_{i=1}^{n} \tilde{\theta}_{i} \dot{\hat{\theta}}_{i} \\
& =\sum_{i=1}^{n}\left(-\frac{n}{\rho T}\left|z_{i}\right|^{2-\rho} \exp \left(\left|z_{i}\right|^{\rho}\right)-\sum_{i=1}^{n} \lambda_{i}\left|z_{i}\right|\right)<0 .
\end{aligned}
$$

\section{Stability Analysis}

The main result of this research is analysed in this section.

Theorem 1. Take into account the closed system consisting of system (1), control laws (28), (40), (47), and (56), and the adaptive laws (29), (41), (48), and (57). If there is a tuning parameter $\lambda_{i}$ such that $(1 / 2) \widetilde{\theta}_{i} \leq \lambda_{i}$, then based on assumptions 1 and 2, we can guarantee that all the closed-loop signals are bounded and the tracking error converges to zero within the predefined time $T$.

Proof

(1) According to (64) and (65), we can know that $z_{i}, \widetilde{\theta}_{i}$ are bounded. The boundedness of $\widehat{\theta}_{i}$ can be deduced from the fact that $\theta_{i}$ is a constant and $\hat{\theta}_{i}$ is bounded. Depend on the boundedness property of $y_{r}$, we can know that the system state $x_{1}$ is bounded. By utilizing the boundedness of $z_{1}$ and constants $q, \underline{h}_{1}, n, \rho, T$, and $\lambda_{1}$, the boundedness property of $\alpha_{1}$ is known. In addition, we already know that $z_{2}$ is bounded, then we can obtain that $x_{2}$ is bounded. Since $\left(\partial \alpha_{1} / \partial z_{1}\right)$ and $\left(\partial \alpha_{1} / \partial \tilde{\theta}_{1}\right)$ are continuous functions with bounded arguments and $\varepsilon_{1}$ is bounded, $\dot{\alpha}_{1}$ and $\sigma_{12}$ are bounded. Considering that $z_{2}$ and $\tilde{\theta}_{2}$ are bounded and $\underline{h}_{2}, q, \bar{h}_{1}$, $n, \rho, T$, and $\lambda_{2}$ are constants, we have $\alpha_{2}$ is bounded. Because $\alpha_{2}$ and $z_{3}$ are bounded, we have $x_{3}$ is bounded. Likewise, we can obtain that $x_{i}, \alpha_{i}$, and $\sigma_{i 2}$ are bounded. Accordingly, we can obtain the boundedness property of all closed-loop signals.

(2) Because of the boundedness property of all the closed-loop signals, we can find a constant $\lambda_{i}$ such that $(1 / 2) \theta_{i} \leq \lambda_{i}$. Then we have

$$
\begin{aligned}
\dot{V}_{n} & \leq \sum_{i=1}^{n}\left(-\frac{n}{\rho T}\left|z_{i}\right|^{2-\rho} \exp \left(\left|z_{i}\right|^{\rho}\right)\right) \\
& =-\frac{n}{\rho T} \sum_{i=1}^{n}\left(\left(z_{i}^{2}\right)^{1-(\rho / 2)} \exp \left(\left(z_{i}^{2}\right)^{(\rho / 2)}\right)\right) .
\end{aligned}
$$

According to Lemma 3, we have 


$$
\begin{aligned}
& -\frac{n}{\rho T} \sum_{i=1}^{n}\left(\left(z_{i}^{2}\right)^{1-(\rho / 2)} \exp \left(\left(z_{i}^{2}\right)^{(\rho / 2)}\right)\right) \\
& \leq-\frac{n}{\rho T} \cdot \frac{1}{n} \sum_{i=1}^{n}\left(z_{i}^{2}\right)^{1-(\rho / 2)}\left(\sum_{i=1}^{n} \exp \left(\left(z_{i}^{2}\right)^{(\rho / 2)}\right)\right) .
\end{aligned}
$$

From Lemma 5, we can derive

$$
\begin{aligned}
& \frac{1}{n} \sum_{i=1}^{n} \exp \left(\left(z_{i}^{2}\right)^{(\rho / 2)}\right) \geq\left(\prod_{i=1}^{n} \exp \left(\left(z_{i}^{2}\right)^{(\rho / 2)}\right)\right)^{(1 / n)} \\
& =\exp \left(\frac{1}{n} \sum_{i=1}^{n}\left(z_{i}^{2}\right)^{(\rho / 2)}\right) .
\end{aligned}
$$

Therefore, based on (67) and (68), one has

$$
\begin{aligned}
\dot{V}_{n} & \leq-\frac{n}{\rho T} \sum_{i=1}^{n}\left(z_{i}^{2}\right)^{1-(\rho / 2)}\left(\prod_{i=1}^{n} \exp \left(\left(z_{i}^{2}\right)^{(\rho / 2)}\right)\right)^{(1 / n)} \\
& \leq-\frac{n}{\rho T} \sum_{i=1}^{n}\left(z_{i}^{2}\right)^{1-(\rho / 2)} \exp \left(\frac{1}{n} \sum_{i=1}^{n}\left(z_{i}^{2}\right)^{(\rho / 2)}\right) .
\end{aligned}
$$

Then, from Lemma 6, we obtain

$$
\dot{V}_{n} \leq-\frac{n}{\rho T}\left(\sum_{i=1}^{n} z_{i}^{2}\right)^{1-(\rho / 2)} \exp \left(\frac{1}{n}\left(\sum_{i=1}^{n} z_{i}^{2}\right)^{(\rho / 2)}\right) \text {. }
$$

Since $0<(\rho / 2)<1$, according to Lemma 1 , we can estimate the stabilization time as

$$
t=T\left(1-\exp \left(-\frac{1}{\beta}\left(2 V_{0}\right)^{(\rho / 2)}\right)\right) \leq T .
$$

Therefore, the tracking error $z_{1}$ will converge to zero within predefined time $T$.

\section{Simulation Example}

The superior performance of the predefined-time controller can be illustrated by the simulation in this section. An uncertain second-order nonlinear system with pure-feedback form is considered as follows:

$$
\left\{\begin{array}{l}
\dot{x}_{1}=0.5 \cos \left(x_{1}\right)+6 x_{2}+0.05 \sin \left(x_{2}\right), \\
\dot{x}_{2}=\cos \left(x_{2}^{2}\right)+12 u+0.5 \sin u+0.1 \sin (t), \\
y=x_{1},
\end{array}\right.
$$

where $\quad f_{1}\left(\bar{x}_{1}, x_{2}\right)=0.5 \cos \left(x_{1}\right)+6 x_{2}+0.05 \sin \left(x_{2}\right)$, $f_{2}\left(\bar{x}_{2}, u\right)=\cos \left(x_{2}^{2}\right)+12 u+0.5 \sin u, \quad$ and $\quad d(t)=0.1$ $\sin (t)$. In the simulation, it is desired that all signals in the closed-loop system are bounded under the proposed control approach, and the system output $y=x_{1}$ can follow the reference trajectory $y_{r}=0.4(\sin (t))+\sin (0.5 t)$.

According to the predefined-time control strategy that has been designed, the virtual controller and actual controller are designed as follows:

$$
\begin{aligned}
\alpha_{1}= & -\frac{1}{\underline{h}_{1}}\left(\frac{n}{\rho T} \operatorname{sig}\left(z_{1}\right)^{1-\rho} \exp \left(\left|z_{1}\right|^{\rho}\right)+\left(\frac{1}{2} \widehat{\theta}_{1}+q+2\right) \operatorname{sign}\left(z_{1}\right)+\lambda_{1} \operatorname{sign}\left(z_{1}\right)\right), \\
u= & -\frac{1}{\underline{h}_{2}}\left(\frac{n}{\rho T} \operatorname{sig}\left(z_{2}\right)^{1-\rho} \exp \left(\left|z_{2}\right|^{\rho}\right)+\left(\frac{1}{2} \widehat{\theta}_{2}+q+3\right) \operatorname{sign}\left(z_{2}\right)\right. \\
& \left.+\left|\sigma_{12}\right| \operatorname{sign}\left(z_{2}\right)+\lambda_{2} \operatorname{sign}\left(z_{2}\right)+z_{1} \bar{h}_{1} \operatorname{sign}\left(z_{2}\right)\right) .
\end{aligned}
$$
forms:

And the adaptive laws are constructed with the following

$$
\begin{aligned}
& \dot{\hat{\theta}}_{1}=\frac{1}{2}\left|z_{1}\right|, \\
& \dot{\hat{\theta}}_{2}=\frac{1}{2}\left|z_{2}\right| .
\end{aligned}
$$

In the simulation, the initial values are assigned as $\left[x_{1}(0), x_{2}(0), \widehat{\theta}_{1}(0), \widehat{\theta}_{2} \quad(0), \sigma 11(0), \sigma 12(0)\right]=[0.5,0.5$, $0.05,0.05,0.05,0.05]^{T}$, where $\sigma_{11}(0)$ and $\sigma_{12}(0)$ represent initial values of the states in finite-time differentiator. Consequently, the design parameters for the virtual controller and actual controller are selected as $\underline{h}_{1}=5, \bar{h}_{1}=$ 10, $\underline{h}_{2}=5, q=10, n=3, \rho=0.3, T=0.8, \quad \lambda_{1}=10.2, \lambda_{2}=$ 10.2 , and the differentiator parameters for the finite-time differentiator are assigned as $k_{1}=2$ and $k_{2}=2.2$.

The results of the simulation can be seen directly in Figures 1 to 3 . Figure 1 gives the system's output $y$ and reference signal $y_{r}$, through which we know that the output $y$ can realize the tracking performance. It can be observed obviously in Figure 1 that $y$ can track the trajectory of the reference signal $y_{r}$ in a predefined time $T=0.8$. From Figure 2, it is obvious that the tracking error $z_{1}$ rapidly approaches 0 . Obviously, the boundedness of states $x_{1}(t)$ and $x_{2}(t)$ can be observed in Figures 1 and 3. The 


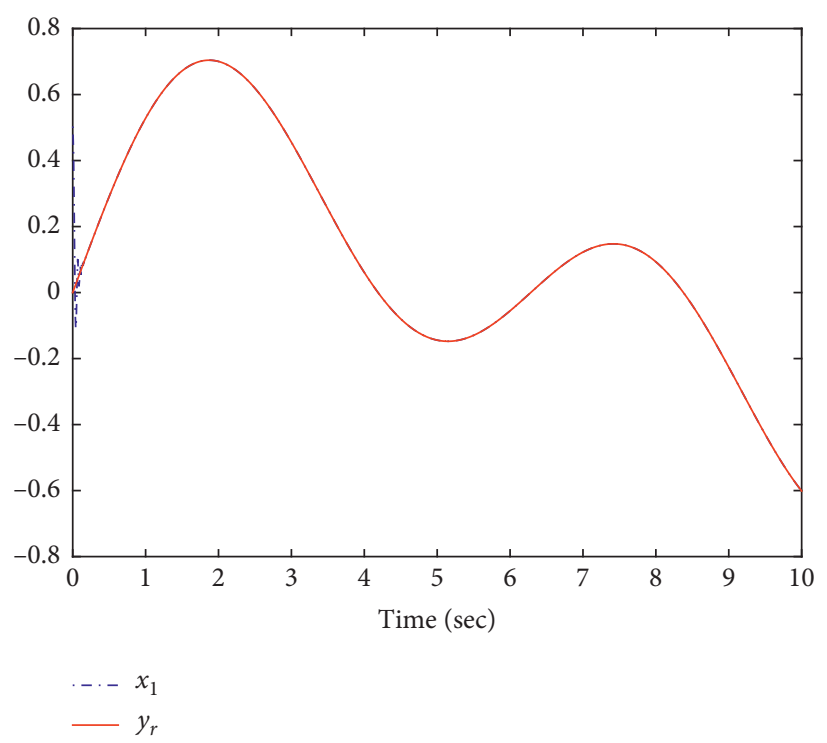

Figure 1: System output $y$ and reference signal $y_{r}$.

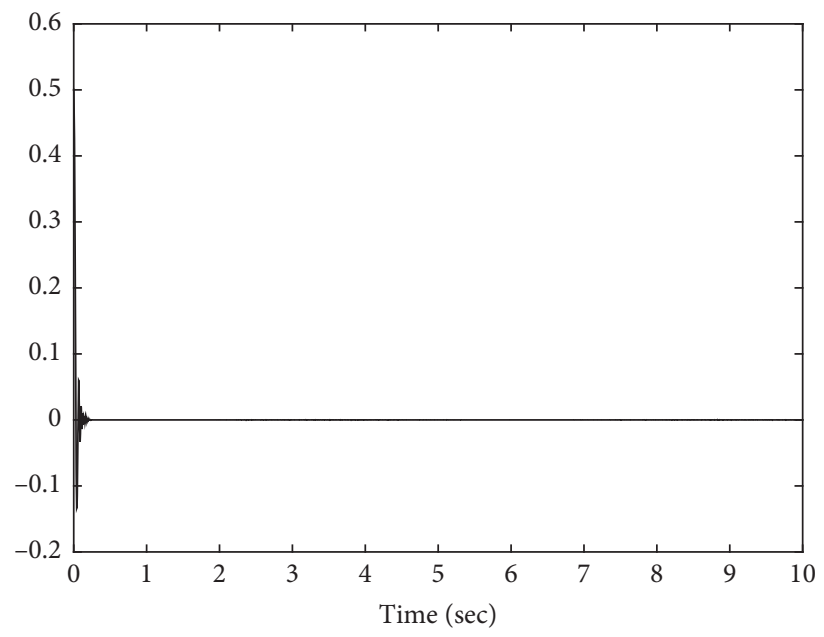

$-z_{1}$

FIGURE 2: The tracking error $z_{1}$.

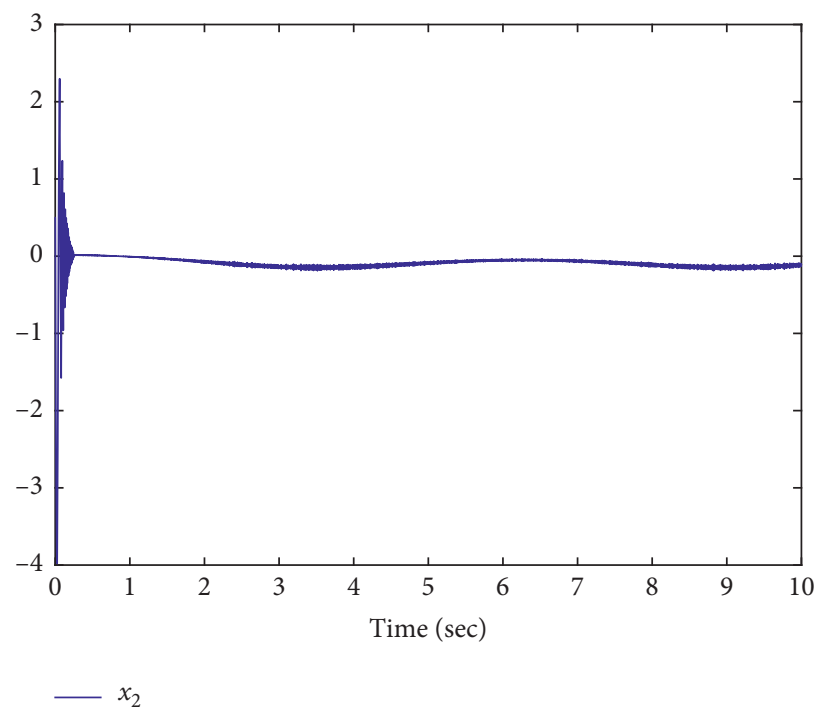

Figure 3: System state $x_{2}$. 
predefined-time controller in this article is effective, which has been illustrated by the simulation in this paper.

\section{Conclusions}

Based on the predefined-time stability theory combined with RBF NN and finite-time differentiator, the adaptive predefined-time controller is designed in this paper for the nonlinear pure-feedback systems with unknown disturbance; simultaneously, it needs to construct the Lyapunov function using the backstepping method. The mean value theorem is used to address the nonaffine problem of the pure-feedback systems, and RBF NN is applied to deal with the unknown nonlinear functions such that the virtual control inputs can be obtained, whose derivatives are further estimated by the finite-time differentiators such that the problem of explosion of complexity can be avoided. The boundedness of all the signals in the closed-loop system can be obtained, and the tracking error can approach zero in a setting time. In the actual situation, the upper bound of the setting time can be arbitrarily designed. Thereby, the adaptive predefined-time controller in this article can be applied to many practical nonlinear pure-feedback systems. However, there are fewer adjustable parameters for the adaptive law $\hat{\theta}_{i}$, which affects the tracking performance of the proposed predefined-time controller. Thereby, we will continue to study how to design an improved adaptive law $\widehat{\theta}_{i}$ to improve the performance of the proposed predefinedtime controller.

\section{Data Availability}

The data used to support the findings of this study are available from the corresponding author upon request.

\section{Conflicts of Interest}

The authors declare that they have no conflicts of interest.

\section{Acknowledgments}

This work was supported in part by the National Natural Science Foundation of China under Grant 61773072 and in part by the Education Department of Liaoning Province under the general project research under Grant no. LJ2020001.

\section{References}

[1] M. Krstic, I. Kanelakopoulos, and P. V. Kokotovic, Nonlinear and Adaptive Control Design, Wiley, New York, NY, USA, 1995.

[2] I. Kanellakopoulos, P. V. Kokotovic, and A. S. Morse, "Systematic design of adaptive controllers for feedback linearizable systems," IEEE Transactions on Automatic Control, vol. 36, no. 11, pp. 1241-1253, 1991.

[3] Y. Sun, B. Chen, C. Lin, H. Wang, and S. Zhou, "Adaptive neural control for a class of stochastic nonlinear systems by backstepping approach," Information Sciences, vol. 369, pp. 748-764, 2016.
[4] D. Yang, X. Li, and J. Qiu, “Output tracking control of delayed switched systems via state-dependent switching and dynamic output feedback," Nonlinear Analysis: Hybrid Systems, vol. 32, pp. 294-305, 2019.

[5] S. S. Ge and C. Cong Wang, "Direct adaptive NN control of a class of nonlinear systems," IEEE Transactions on Neural Networks, vol. 13, no. 1, pp. 214-221, 2002.

[6] S. S. Ge, G. Y. Li, J. Zhang, and T. H. Lee, "Direct adaptive control for a class of MIMO nonlinear systems using neural networks," IEEE Transactions on Automatic Control, vol. 49, no. 11, pp. 2001-2006, 2004.

[7] Y. C. Yeong-Chan Chang, "An adaptive H/sup $\infty /$ tracking control for a class of nonlinear multiple-input multipleoutput (MIMO) systems," IEEE Transactions on Automatic Control, vol. 46, no. 9, pp. 1432-1437, 2001.

[8] M. M. Polycarpou, "Stable adaptive neural control scheme for nonlinear systems," IEEE Transactions on Automatic Control, vol. 41, no. 3, pp. 447-451, 1996.

[9] X. Li, D. O’Regan, and H. Akca, "Global exponential stabilization of impulsive neural networks with unbounded continuously distributed delays," IMA Journal of Applied Mathematics, vol. 80, no. 1, pp. 85-99, 2015.

[10] M. Chen and S. S. Ge, “Adaptive neural output feedback control of uncertain nonlinear systems with unknown hysteresis using disturbance observer," IEEE Transactions on Industrial Electronics, vol. 62, no. 12, pp. 7706-7716, 2015.

[11] H. Wang, P. Shi, H. Li, and Q. Zhou, "Adaptive neural tracking control for a class of nonlinear systems with dynamic uncertainties," IEEE Transactions on Cybernetics, vol. 47, no. 10, pp. 3075-3087, 2017.

[12] X. Zhao, X. Wang, S. Zhang, and G. Zong, "Adaptive neural backstepping control design for A class of nonsmooth nonlinear systems," IEEE Transactions on Systems, Man, and Cybernetics: Systems, vol. 49, no. 9, pp. 1820-1831, 2019.

[13] J. Na, M. N. Mahyuddin, G. Herrmann, X. Ren, and P. Barber, "Robust adaptive finite-time parameter estimation and control for robotic systems," International Journal of Robust and Nonlinear Control, vol. 25, no. 16, pp. 3045-3071, 2015.

[14] H. Du, W. Zhu, G. Wen, and D. Wu, "Finite-time formation control for a group of quadrotor aircraft," Aerospace Science and Technology, vol. 69, pp. 609-616, 2017.

[15] W. He, S. Zhang, and S. S. Ge, "Adaptive control of a flexible crane system with the boundary output constraint," IEEE Transactions on Industrial Electronics, vol. 61, no. 8, pp. 4126-4133, 2014.

[16] S. S. Ge and C. Wang, "Adaptive NN control of uncertain nonlinear pure-feedback systems," Automatica, vol. 38, no. 4, pp. 671-682, 2002.

[17] C. Wang, D. J. Hill, S. S. Ge, and G. Chen, "An ISS-modular approach for adaptive neural control of pure-feedback systems," Automatica, vol. 42, no. 5, pp. 723-731, 2006.

[18] B. Ren, S. S. Ge, C. Y. Su, and T. H. Lee, "Adaptive neural control for a class of uncertain nonlinear systems in purefeedback form with hysteresis input," IEEE Transactions on Systems, Man, and Cybernetics, Part B (Cybernetics), vol. 39, no. 2, pp. 431-443, 2009.

[19] P. Dorato, Short Time Stability in Linear Time-Varying Systems, Polytechnic Institute of Brooklyn, Microwave Research Institute, New York, NY, USA, 1961.

[20] L. Weiss and E. Infante, "Finite time stability under perturbing forces and on product spaces," IEEE Transactions on Automatic Control, vol. 12, no. 1, pp. 54-59, 1967.

[21] Y. Liu, X. Liu, Y. Jing, X. Chen, and J. Qiu, "Direct adaptive preassigned finite-time control with time-delay and quantized 
input using neural network," IEEE Transactions on Neural Networks and Learning Systems, vol. 31, no. 4, pp. 1222-1231, 2020.

[22] M. Golestani, S. Mobayen, and F. Tchier, "Adaptive finitetime tracking control of uncertain non-linear $\mathrm{n}$-order systems with unmatched uncertainties," IET Control Theory \& Applications, vol. 10, no. 14, pp. 1675-1683, 2016.

[23] X. Huang, W. Lin, and B. Yang, "Global finite-time stabilization of a class of uncertain nonlinear systems," Automatica, vol. 41, no. 5, pp. 881-888, 2005.

[24] H. Wang, P. X. Liu, X. Zhao, and X. Liu, "Adaptive fuzzy finite-time control of nonlinear systems with actuator faults," IEEE Transactions on Cybernetics, vol. 50, no. 5, pp. 1786$1797,2020$.

[25] W. Lv, F. Wang, and Y. Li, "Finite-time adaptive fuzzy outputfeedback control of MIMO nonlinear systems with hysteresis," Neurocomputing, vol. 296, pp. 74-81, 2018.

[26] A. Polyakov, D. Efimov, and W. Perruquetti, "Finite-time and fixed-time stabilization: implicit Lyapunov function approach," Automatica, vol. 51, pp. 332-340, 2015.

[27] M. Chen, H. Wang, and X. Liu, "Adaptive fuzzy practical fixed-time tracking control of nonlinear systems," IEEE Transactions on Fuzzy Systems, vol. 29, no. 3, pp. 664-673, 2021.

[28] H. Wang, K. Xu, and J. Qiu, "Event-triggered adaptive fuzzy fixed-time tracking control for a class of nonstrict-feedback nonlinear systems," IEEE Transactions on Circuits and Systems I: Regular Papers, vol. 68, no. 7, pp. 3058-3068, 2021.

[29] J. Hu, G. Sui, X. Lv, and X. Li, "Fixed-time control of delayed neural networks with impulsive perturbations," Nonlinear Analysis: Modelling and Control, vol. 23, no. 6, pp. 904-920, 2018.

[30] S. Ling, H. Wang, and P. X. Liu, "Fixed-time adaptive eventtriggered tracking control of uncertain nonlinear systems," Nonlinear Dynamics, vol. 100, no. 4, pp. 3381-3397, 2020.

[31] X. Jin, “Adaptive fixed-time control for MIMO nonlinear systems with asymmetric output constraints using universal barrier functions," IEEE Transactions on Automatic Control, vol. 64, no. 7, pp. 3046-3053, 2019.

[32] J. D. Sánchez-Torres, D. Gómez-Gutiérrez, and E. López, "A class of predefined-time stable dynamical systems," IMA Journal of Mathematical Control and Information, vol. 35, pp. i1-i29, 2018.

[33] J. K. Ni, L. Liu, Y. Tang, and C. X. Liu, "Predefined-time consensus tracking of second-order multiagent systems," IEEE Transactions on Systems, Man, and Cybernetics: Systems, vol. 51, no. 4, pp. 2550-2560, 2019.

[34] J. D. Snchez-Torres, D. Gmez-Gutirrez, and A. G. Loukianov, "Predefined-time backstepping control for tracking aclass of mechanical systems," in 20th IFAC World Congress, vol. 50, no. 1, pp. 1680-1685, Toulouse, France, July 2017.

[35] J. Ni and P. Shi, "Global predefined time and accuracy adaptive neural network control for uncertain strict-feedback systems with output constraint and dead zone," IEEE Transactions on Systems, Man, and Cybernetics: Systems, vol. 80, pp. 1-16, 2020.

[36] A. Ferrara and G. P. Incremona, "Predefined-time output stabilization with second order sliding mode generation," IEEE Transactions on Automatic Control, vol. 66, no. 3, pp. 1445-1451, 2021.

[37] H. Wang, S. Liu, and W. Bai, "Adaptive neural tracking control for non-affine nonlinear systems with finite-time output constraint," Neurocomputing, vol. 397, no. 15, pp. 60-69, 2020.
[38] X. F. Zhang, G. Feng, and Y. H. Sun, "Finite-time stabilization by state feedback control for a class of time-varying nonlinear systems," Automatica, vol. 48, no. 14, pp. 499-504, 2012.

[39] E. Jimenez-Rodriguez, J. D. Snchez-Torres, and A. G. Loukianov, "On optimal predefined-time stabilization," International Journal of Robust and Nonlinear Control, vol. 27, no. 17, pp. 3620-3642, 2017.

[40] J. Bao, H. Wang, and P. X. Liu, "Finite-time synchronization control for bilateral teleoperation systems with asymmetric time-varying delay and input dead zone," IEEE/ASME Transactions on Mechatronics, vol. 255, 2018.

[41] X. Li, J. Shen, and R. Rakkiyappan, "Persistent impulsive effects on stability of functional differential equations with finite or infinite delay," Applied Mathematics and Computation, vol. 329, pp. 14-22, 2018.

[42] G. Hardy and J. Littlewood, Inequalities, Cambridge University Press, London, UK, 1988.

[43] A. Levant, "Robust exact differentiation via sliding mode technique," Automatica, vol. 34, no. 3, pp. 379-384, 1998. 